\title{
Problemas relacionados com o uso de testes de comparação de médias em artigos científicos
}

\author{
Juliano Garcia Bertoldo \\ Jefferson Luís Meirelles Coimbra* \\ Altamir Frederico Guidolin \\ Adelar Mantovani \\ Naine Martins do Vale \\ Universidade do Estado de Santa Catarina (UDESC), Centro de Ciências Agroveterinárias (CAV) \\ Instituto de Melhoramento e Genética Molecular (IMEGEM) \\ Av. Camões 2090, CEP 88520000, Lages - SC, Brasil \\ *Autor para correspondência \\ coimbrajefferson@cav.udesc.br
}

Submetido em 23/08/2007

Aceito para publicação em 28/12/2007

\section{Resumo}

Este trabalho teve como objetivo verificar quais as principais dificuldades dos pesquisadores com relação ao uso de testes de comparação de médias, bem como propor alternativas para uma melhor inferência estatística. Para uma aplicação correta dos testes estatísticos é fundamental o conhecimento por parte do pesquisador, do delineamento experimental, dos tipos de fatores, das variáveis respostas e dos tratamentos que irão constituir o seu trabalho. Foram revisados duzentos e noventa e dois (292) trabalhos científicos na área de fitotecnia, publicados num periódico da área das Ciências Agrárias, Qualis A nacional, no período compreendido entre 2000 e 2006. Para tanto, os artigos foram classificados quanto à utilização de testes de comparação de médias em: $i$ ) apropriado; ii) parcialmente apropriado; iii) inapropriado. Existe dificuldade na escolha do procedimento correto em relação ao tipo de fator experimental estudado, sendo que dos trabalhos que empregaram mais de um fator, (75\%) foram classificados como inapropriados devido ao abuso dos testes de comparações de médias. Os demais, que representam $3 \%$ e $22 \%$, foram classificados como parcialmente apropriados e apropriados, respectivamente.

Unitermos: fatores qualitativos e quantitativos, comparação de médias, contrastes, regressão

\section{Abstract}

Difficulties related to the use of tests of comparison of averages in scientific articles. The objective of this work was to verify the researcher's main difficulties in the use of means comparison tests, as well as to propose alternatives for a better statistical inference. For a correct application of the statistical tests it is fundamental for the researcher to know the experimental design, the factors, the variables and the treatments that will constitute the work, because these are decisive in the choice of the statistical test. Two hundred and ninety-two scientific papers from plant sciences were revised. These papers were published in the Brazilian Qualis A journal, from 2000 until 2006. The papers were classified by their use of means comparison tests as i) appropriate, ii) 
partially appropriate, and iii) inappropriate. There were difficulties involved in choosing the correct statistical procedure in relation to the experimental design used. The papers in which more than one factor was studied (75\%) were classified as inadequate due to their abuse of the means comparison tests. On the other hand, 3\% and $22 \%$ were classified as partially appropriate and appropriate, respectively.

Key word: qualitative and quantitative factors, means comparison, contrasts, regression

\section{Introdução}

Diversos testes estatísticos permitem aos pesquisadores inferir sobre os resultados experimentais. No entanto, para a aplicação correta dos testes estatísticos, é de fundamental importância que o pesquisador tenha conhecimento sobre quais os tipos de fatores, de variáveis respostas, de tratamentos e do delineamento experimental que irão constituir o seu trabalho. Segundo Silva (1999), a fundamentação teórica do delineamento de tratamento de um experimento, tal como os tipos de fatores envolvidos, é fundamental para as inferências do pesquisador. Ainda, de acordo com o mesmo autor, a estrutura dos fatores experimentais, a estrutura dos fatores de unidade e a relação entre essas duas estruturas, determinada pela casualização, constituem a estrutura do experimento ou o delineamento experimental. Assim, no intuito de explicar a resposta dos dados obtidos de maneira mais detalhada, vários procedimentos podem ser utilizados, como por exemplo, o ajuste de funções de resposta, usando as técnicas de regressão (Jones, 1984), os conjuntos de contrastes planejados entre as médias ou grupos de médias (Gill, 1978) e os procedimentos de comparação múltiplas de médias (Chew, 1976), sendo que, os testes de comparação de médias freqüentemente são os mais utilizados pelos pesquisadores (Aflakpui, 1995).

Porém, apesar de uma diversidade de testes estatísticos à disposição dos pesquisadores, a maioria os utiliza de modo incorreto. Em seus estudos, Petersen (1977) verificou que $40 \%$ dos autores pesquisados utilizaram em suas análises, algum tipo de teste de comparação de médias e que destes, $40 \%$ os utilizaram de forma inteiramente inapropriada quanto ao tipo dos dados envolvidos. De acordo com o mesmo autor, os testes de comparação de médias (Tukey, Bonferroni, etc.) são apropriados para situações em que os tratamentos são níveis de fator qualitativo e não relacionados. Porém, em muitos experimentos os procedimentos de comparação de médias são aplicados em fatores quantitativos, onde o correto seria a utilização de análise de regressão.

O procedimento apropriado para discriminar a variação entre os contrastes de interesse deve ser realizado de acordo com o objetivo experimental e com o tipo de fator em estudo. Por exemplo, se o pesquisador desejar medir os efeitos dos tratamentos de fatores quantitativos (doses de um fertilizante, densidade populacional, concentrações de hormônios, etc.), o procedimento apropriado é o ajuste de uma equação de regressão. Por outro lado, se pesquisador objetivar inferir sobre os efeitos de fatores qualitativos não estruturados (variedades, tipos de solo, local, etc.), o apropriado é a utilização de testes de comparação de médias, como por exemplo, o de Tukey.

Portanto, este trabalho teve como objetivo verificar se os testes de comparação de médias foram corretamente aplicados em trabalhos científicos, sugerindo alternativas viáveis no intuito de aprimorar a interpretação dos resultados, bem como demonstrar quais os principais erros mediante exemplos ilustrativos.

\section{Material e Métodos}

Foram revisadas duzentos e noventa e duas (292) publicações científicas de um periódico Qualis A nacional, no período compreendido entre 2000 e 2006, somente na área de Fitotecnia, utilizando deste total, cento e noventa e quatro (194) trabalhos para discussão. Os trabalhos que envolviam análise multivariada, ou aqueles que não utilizaram testes de comparação de médias não foram revisados, uma vez que este não era o objetivo do trabalho.

Os artigos foram classificados quanto ao emprego de testes de comparação de médias em: i) apropriado; ii) parcialmente apropriado e; iii) inapropriado. Foi 
atribuído o critério apropriado quando utilizada análise de regressão para fatores quantitativos e testes de comparação de médias aos tratamentos de natureza qualitativa e não relacionados, desde que utilizados de modo coerente conforme os objetivos propostos pelo trabalho e da estrutura de fatores. A classificação de parcialmente apropriado foi aplicada aos testes de comparações de médias que compararam os tratamentos entre si, no entanto o procedimento adequado seria a realização de contrastes previamente planejados, ou seja, aplicação de contrastes ortogonais para fatores qualitativos estruturados. Finalmente, o critério inapropriado, envolveu fatores quantitativos, com a utilização de algum teste de comparação de médias, e a não verificação do efeito da interação em experimentos fatoriais, ou seja, com mais de um fator.

Para exemplificação dos resultados, os trabalhos foram divididos em três grupos: grupo I para trabalhos apropriados; grupo II para trabalhos parcialmente apropriados e grupo III para trabalhos inapropriados. Além disso, foram ilustrados dois exemplos no intuito de colaborar com os pesquisadores, onde estes apresentaram maiores dificuldades: aplicação de teste de comparação de médias e análise de regressão para fatores quantitativos e contrastes ortogonais.

\section{Resultados e Discussão}

Para Banzatto e Kronka (2006), um dos objetivos da estatística é a tomada de decisões a respeito da população, com base na observação das amostras, ou seja, a obtenção de conclusões válidas para todos os parâmetros populacionais, a partir de amostras retiradas dessa população. Ao se tentar chegar a decisões é conveniente a formulação de hipóteses ou de conjecturas acerca das populações interessadas (Spiegel, 1993). A análise de variância preliminar permite ao pesquisador testar as hipóteses, ou seja, rejeitar $\mathrm{H}_{\mathrm{o}}$ ou não rejeitar $\mathrm{H}_{\mathrm{o}}$ (hipótese de nulidade). Portanto, permite verificar se existe ou não diferenças entre os parâmetros estudados. A análise de variância é uma técnica que consiste na decomposição da variância total e dos graus de liberdade em partes atribuídas a causas conhecidas e independentes, ou seja, fatores controlados; e a uma porção residual de origem desconhecida e natureza aleatória ou fatores não controlados (Banzatto e Kronka, 2006). De acordo com a estrutura de um experimento, os graus de liberdade devem ser desdobrados no intuito de verificar o efeito da interação entre os fatores, quando esta for significativa. Portanto, em experimentos fatoriais, o primeiro passo é analisar o efeito da interação entre os vários fatores (Petersen, 1977).

De forma sucinta, um experimento pode apresentar um ou mais fatores, sendo denominado, respectivamente, de unifatorial e fatorial. De modo geral, os fatores podem ser classificados em qualitativos e/ou quantitativos. Os fatores qualitativos podem ser divididos em dois subgrupos: i) estruturados e ii) não estruturados. Fatores qualitativos estruturados são aqueles cujos níveis podem ser classificados em grupos das quais as comparações constituem o objetivo do trabalho. Os estudos dos fatores qualitativos não estruturados apresentam como objetivo comparações entre todos os níveis do fator experimental, ou seja, todos contra todos (Chew, 1976). Os fatores qualitativos podem ser expressos por uma variável nominal, denominados específicos (ex. cultivares), de escala ordinal, classificados como ordenados (ex. época de plantio - cedo, intermediário, tardio), ou ainda aleatória, chamados de amostrados, cujos níveis utilizados no experimento não são de interesse específico, mas são escolhidos, por um processo supostamente aleatório (ex. local), enquanto que os quantitativos são expressos numa variável intervalar ou racional (ex. doses de um fertilizante).

De acordo com a tabela 1, pode ser observado que o percentual de trabalhos classificados como apropriado quanto ao uso de testes de comparação de médias ao tipo de fator em estudo para a categoria unifatorial foi de 55\% (37 artigos), enquanto para a categoria fatorial foi de $22 \%$ (28 artigos); já o percentual de trabalhos classificados como inapropriados para as categorias unifatorial e fatorial foi de 24\% (16 artigos) e $75 \%$ (94 artigos), respectivamente. 
TABELA 1: Número de artigos revisados quanto ao número de fatores (um fator ou mais de um fator) quanto ao tipo de fator ((qualitativo não estruturado (Qlne), qualitativo estruturado (Qle), quantitativo (Qt)) e quanto ao uso (apropriado, parcialmente apropriado e inapropriado), editados numa revista Qualis A, no período de 2000 a 2006.

\begin{tabular}{|c|c|c|c|c|c|c|c|c|}
\hline \multirow{2}{*}{\multicolumn{2}{|c|}{ Classificação }} & \multicolumn{5}{|c|}{ Unifatorial } & \multirow{2}{*}{ Total } & \multirow{2}{*}{$\%$} \\
\hline & & \multicolumn{2}{|c|}{ QIne } & \multicolumn{2}{|l|}{ Qle } & Qt & & \\
\hline \multicolumn{2}{|l|}{ Apropriado } & \multicolumn{2}{|c|}{22} & \multicolumn{2}{|l|}{1} & 14 & 37 & 55 \\
\hline \multicolumn{2}{|c|}{ Parcialmente Apropriado } & \multicolumn{2}{|c|}{0} & \multicolumn{2}{|l|}{12} & 2 & 14 & 21 \\
\hline \multicolumn{2}{|l|}{ Inapropriado } & \multicolumn{2}{|c|}{7} & \multicolumn{2}{|l|}{5} & 4 & 16 & 24 \\
\hline \multicolumn{2}{|l|}{ Total } & \multicolumn{2}{|c|}{29} & 18 & & 20 & 67 & \multirow[b]{2}{*}{100} \\
\hline \multicolumn{2}{|l|}{$\%$} & \multicolumn{2}{|c|}{43} & 27 & & 30 & & \\
\hline & & & Fatorial & & \multirow[b]{2}{*}{ QI x Qt x Qt } & \multirow[b]{2}{*}{ Total } & \multirow[b]{2}{*}{$\%$} \\
\hline Classificação & ${ }^{1}$ QI x QI & Q1 x Qt & ${ }^{2} \mathbf{Q t} \times \mathbf{Q t}$ & QI x Q1 x Q1 & Q1 x Q1 x Qt & & & \\
\hline Apropriado & 10 & 7 & 6 & 3 & 1 & 1 & 28 & 22 \\
\hline P. Apropriado & 2 & 2 & 0 & 0 & 0 & 0 & 4 & 3 \\
\hline Inapropriado & 32 & 23 & 22 & 4 & 10 & 3 & 94 & 75 \\
\hline Total & 44 & 32 & 28 & 7 & 11 & 4 & 126 & \\
\hline$\%$ & 35 & 25 & 22 & 6 & 9 & 3 & & 100 \\
\hline
\end{tabular}

${ }^{1} \mathrm{Q} \mathrm{l}=$ qualitativo. ${ }^{2} \mathrm{Qt}=$ quantitativo.

Muitas vezes, na experimentação agrícola, os experimentos são conduzidos por meio de experimentos fatoriais, onde os níveis de um fator ocorrem em combinação com os níveis do outro fator. $\mathrm{O}$ arranjo fatorial permite investigar as interações entre os fatores, além de estimar a variância do erro experimental com maior precisão, aumentando o poder dos testes estatísticos (Wechsler, 1998).

Os resultados evidenciam a dificuldade por parte dos pesquisadores na análise dos dados, principalmente em experimentos unifatoriais com fatores quantitativos e em experimentos fatoriais. Nos experimentos fatoriais revisados e classificados como inapropriados, os autores não consideraram o efeito da interação significativa, realizando testes de comparação de médias, como o teste de Tukey, por exemplo, de forma separada para cada fator. O uso de teste de comparação de médias nessas circunstâncias é um abuso comum dessa técnica (Chew, 1976). Portanto, em experimentos fatoriais, o primeiro passo é analisar o efeito das interações entre os vários fatores (Petersen, 1977). Para detectar as interações, o apropriado é uma análise de variância preliminar. $\mathrm{Na}$ estatística experimental, especificamente quando se faz a análise de variância, os testes de hipóteses têm sido amplamente utilizados para se concluir a respeito das fontes de variação consideradas no modelo estatístico. Nesse caso, sendo o teste $F$ significativo para o efeito da interação, os graus de liberdade devem ser rearranjados de modo a comparar os níveis de um fator dentro dos níveis do outro (Cardellino e Siewerdt, 1992). Sendo assim, fixando um fator e variando dentro dos níveis do outro fator, se realiza o que é conhecido por teste de efeitos simples (Winer, 1971). Esse procedimento de variar um fator por vez se aplica quando o objetivo é estabelecer uma lei fundamental, o que conduziria ao conhecimento detalhado de efeito de um fator quando os outros são mantidos constantes (Hinkelmann e Kempthorne, 1994).

A tabela 2 evidencia a utilização de testes de comparação de médias, em experimentos unifatoriais e fatoriais, nos artigos revisados. Nos trabalhos unifatoriais, $48 \%, 26 \%$ e $26 \%$ dos trabalhos foram classificados quanto ao uso de forma apropriada, parcialmente apropriada e inapropriada, respectivamente. Nos experimentos fatoriais, $79 \%$ dos trabalhos revisados foram considerados inapropriados com relação aos testes de comparações de médias, enquanto $17 \%$ apropriados e $4 \%$ parcialmente apropriados.

Esses resultados estão de acordo aos obtidos por Cardellino e Siewerdt (1992) e Santos et al. (1998), que classificaram como incorreta a maioria dos trabalhos ana- 
lisados quanto ao uso dos testes de comparação múltipla de médias, respectivamente, na Revista da Sociedade Brasileira de Zootecnia (Qualis A Internacional) e na Pesquisa Agropecuária Brasileira (Qualis A Internacional). Por outro lado, os resultados são opostos aos obtidos tanto por Lúcio et al. (2003) quanto por Bezerra Neto et al. (2002), que revisaram trabalhos publicados em periódicos nacionais, onde $63 \%$ e $65,6 \%$ destes foram classificados como apropriados quanto ao uso dos testes de comparação de médias, respectivamente.

De acordo com os resultados, foi verificado que a maior dificuldade dos pesquisadores está relacionada com o desdobramento da interação quando significativa. O desconhecimento do procedimento de aplicação e a inabilidade quanto à interpretação dos resultados são alguns exemplos das principais causas da má aplicação dos testes de comparação de médias.

De acordo com o tipo de fator que o trabalho abrange, alguns procedimentos são apontados pela figura 1 para a inferência do pesquisador nos resultados obtidos.
TABELA 2: Classificação dos trabalhos unifatorial (um fator somente) e fatorial (mais de um fator) editados numa revista Qualis A (2000 a 2006), quanto ao uso de testes de comparação de médias.

\begin{tabular}{lccc}
\multicolumn{1}{c}{ Teste } & \multicolumn{3}{c}{ Unifatorial } \\
Duncan & Apropriado & P. Apropriado & Inapropriado \\
Tukey & 7 & 6 & 12 \\
Scott \& Knott & 9 & 5 & 0 \\
Dms & 2 & 1 & 0 \\
Dunett & 0 & 0 & 0 \\
\hline Total & 23 & 0 & 1 \\
\hline & & 12 & 12 \\
\cline { 2 - 4 } \multicolumn{1}{c}{ Teste } & Apropriado & P. Apropriado & Inapropriado \\
\hline Duncan & 4 & 2 & 37 \\
Tukey & 11 & 1 & 30 \\
Scott \& Knott & 1 & 1 & 4 \\
Dms & 1 & 0 & 4 \\
Dunnett & 0 & 0 & 76 \\
\hline Total & 16 & 4 & \\
\hline
\end{tabular}

TUKEY

- Interesse em comparar

todos os pares de médias

entre si. Exige dados

balanceados.

SCHEFFÉ

- Quando os contrastes são

definidos a posteriori. Não

exige dados balanceados

NÃO ESTRUTURADOS

- Comparações entre todos os

níveis do fator experimental

BONFERRONI

- Aplicado para

contrastes não

ortogonais. Não exige

dados balanceados

DUNNETT

- Interesse em comparar

os tratamentos com uma

testemunha

ESTRUTURADOS

- Comparação entre grupos

constituem o objetivo do trabalho

CONTRASTES

- Contrastes ortogonais

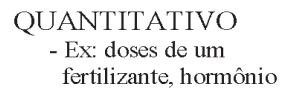

REGRESSÃO

- Permite estudar qualquer nível intermediário

de um fator em questão, mesmo que não esteja incluído

diretamente no estudo

FIGURA 1: Opção para escolha do teste em relação ao tipo de fator. 


\section{I - Exemplos:}

\section{Grupo I (Apropriado)}

Unifatorial: como primeiro exemplo, foi selecionado um artigo publicado no volume 35 , n.6, p.1281-1286, nov - dez, 2005, que tinha como objetivo avaliar o desempenho de plantas de "Folha Murcha" enxertadas em seis portaenxertos no Norte do Estado do Paraná. Como o fator de estudo é qualitativo (porta-enxertos) e se objetivava comparar todos os tratamentos entre si, a comparação entre médias é o procedimento apropriado. Assim, os autores utilizaram o teste de Duncan para inferir sobre os resultados obtidos de modo apropriado. No entanto, deve ser ressaltado que com três ou mais médias sendo comparadas, a teoria do teste de Duncan é errada, pois o nível de significância global não é mantido (Gill, 1978).

Fatorial: No trabalho publicado no volume 34, n.2, p.379-383, mar - abr, 2004, que tinha como objetivo avaliar a adubação nitrogenada sobre atributos qualitativos dos grãos e das sementes em aveia branca, a aplicação do teste de comparação de médias foi adequada, bem como a utilização de análise de regressão. Como este trabalho apresentou tanto fatores qualitativos (cultivares), quanto quantitativos (níveis de adubação nitrogenada), foi necessário ajustar uma equação de regressão para o fator quantitativo. Entretanto, como não houve interação entre os fatores, foi possível analisar separadamente o efeito principal de cada fator independentemente do outro. Assim, aplicou-se teste de comparação de médias (teste de Duncan) para o fator qualitativo e análise de regressão para o fator quantitativo.

\section{Grupo II (Parcialmente Apropriado)}

Unifatorial: $O$ trabalho que tinha como objetivo caracterizar alguns substratos e algumas misturas (combinações) dos substratos, em termos químicos e físicos, e comparar os resultados para a formulação de substratos para o cultivo de mudas frutíferas e de flores em recipientes, utilizou o teste de Duncan de maneira inadequada. Os tratamentos avaliados no trabalho foram: solo + areia (1:1); solo + areia + CAC (1:1:1); solo + areia + RDCA (1:1:1); turfa SCv + CAC (2:1) e turfa $\mathrm{SCv}+\mathrm{RDCA}(2: 1)$.
Como o objetivo foi de equiparar tratamentos específicos, a comparação por contrastes não ortogonais, como teste de comparação de médias, não é o procedimento mais apropriado, uma vez que, para se obter dados mais informativos, é necessário relacionar os tratamentos por contrastes para verificar qual dos fatores (solo, areia, CAC, RDCA ou turfa) está realmente fazendo a diferença. Por exemplo, realizando um contraste entre os tratamentos solo + areia (1:1) e solo + areia + RDCA (1:1:1), o pesquisador poderia conhecer se o RDCA foi o fator que fez diferença no resultado final de avaliação. Como esses dois tratamentos se diferenciaram significativamente para as características físicas, com a aplicação de contrastes ortogonais, os resultados poderiam ser melhores explorados, identificando corretamente qual o fator que está influenciando positivamente (aumentando a média do tratamento) ou negativamente (diminuindo a média do tratamento), porém significativamente, podendo ser testado tanto pelo teste $F$ ou pelo teste $t$. O teste de Duncan, nesse caso, não permite identificar se é o RDCA que propicia ou não vantagem em termos físicos, uma vez que envolve apenas duas médias. Como alternativa, portanto, poderiam ser realizados alguns contrastes ortogonais entre os tratamentos, para se testar o efeito das diferentes combinações.

\section{Grupo III (Inapropriados)}

Unifatorial: o trabalho onde o objetivo foi determinar o efeito da idade fisiológica das mudas de alface no momento do transplante sobre o crescimento e desenvolvimento de plantas de lavoura representa um exemplo da má aplicação de teste de comparação de médias. Os tratamentos consistiram de quatro datas de semeadura $(5,11,19$ e 26 de julho). Como os tratamentos são épocas de semeadura $\left(\mathrm{T}_{1}, \mathrm{~T}_{2}, \mathrm{~T}_{3} \mathrm{e} \mathrm{T}_{4}\right)$, portanto, fator quantitativo, o apropriado seria o ajuste de uma curva de regressão polinomial para os dados. Nesse trabalho os autores submeteram os resultados ao teste de Tukey. De acordo com Chew (1976), se o coeficiente de regressão é significativo, nenhum procedimento de comparação múltipla de médias é necessário, pois todos os efeitos de tratamentos (inclusive os níveis intermediários não utilizados diretamente no experimento) são significativamente diferentes. 
Fatorial: A aplicação de teste de comparação de médias no trabalho que tinha como objetivo determinar as melhores densidades de peixes e época de colocação de alevinos na área que proporcionem as melhores produções no consórcio de arroz irrigado e peixes foi inapropriada. Os tratamentos foram constituídos por dois fatores quantitativos (três densidades de povoamento de peixes e três épocas de colocação dos alevinos) e um fator qualitativo (dois anos agrícolas). Como o experimento apresentou mais de um fator, o primeiro passo seria testar a significância da interação entre os fatores. Os autores comentam que o efeito da interação foi significativo entre os anos agrícolas e épocas de colocação de alevinos com relação à sobrevivência dos mesmos. Ainda assim, utilizaram teste de comparação de médias para todos os efeitos avaliados (efeito principal e as interações). Como alternativa, uma vez que a interação entre os fatores foi significativa, seria apropriado decompor os graus de liberdade da interação e dos graus de liberdade dos efeitos envolvidos. Ainda, o apropriado seria ajustar equações de regressão para a interação entre os anos agrícolas e épocas de colocação dos alevinos e para a densidade de povoamento de peixes.

\section{II - Ilustração}

A partir de dados experimentais obtidos no ano de 2007, pelo grupo de pesquisa IMEGEM do CAV/ UDESC, foram realizadas duas ilustrações, no intuito de demonstrar algum dos erros evidenciados durante a revisão dos artigos científicos previamente discutidos nesse artigo. Assim sendo, foram ilustrados os procedimentos inadequados para a análise dos dados, de acordo com o tipo de fator estudado, e posteriormente, apresentado o modo adequado para a avaliação dos resultados.

Exemplo 1. No primeiro exemplo é enfatizado um experimento constituído por um fator quantitativo (doses de nitrogênio com três níveis). $\mathrm{O}$ objetivo foi avaliar a resposta de três doses de $\mathrm{N}\left(0-50-100 \mathrm{~kg} \cdot \mathrm{ha}^{1}\right)$ no peso de grãos de feijão. Para avaliar qual dose propiciou um melhor peso de grãos, foi aplicado (de maneira inapropriada) o teste de Tukey, e posteriormente ajustada uma equação de regressão para o efeito das doses de nitrogênio.
Nesse primeiro exemplo, o objetivo principal é demonstrar como a análise de regressão é mais explicativa, conduzindo o pesquisador a resultados mais coerentes. Por meio da tabela 3 pode ser verificado que o efeito do fator doses de nitrogênio foi significativo pelo teste $F$.

TABELA 3: Resumo da análise de variância para o peso de grãos feijão. Lages, 2007.

\begin{tabular}{lcc}
\multicolumn{1}{c}{ F.V. } & G.L. & Q.M. \\
Doses de N & 2 & $257,80^{*}$ \\
Erro & 15 & 26,70 \\
\hline Total & 17 & - \\
C.V.\% & - & 2,31 \\
\hline
\end{tabular}

*Significativo ao nível de $5 \%$ de significância pelo teste $F$.

De acordo com a tabela 4, o maior peso dos grãos (g) foi obtido com a dose $50 \mathrm{~kg}$.ha ${ }^{1}$, pelo teste de Tukey. No entanto, conforme a figura 2, ao se ajustar uma equação de regressão, pode ser verificado que o maior peso dos grãos é obtido com a dose 67 kg.ha ${ }^{1}$. Sendo assim, o teste de comparação de médias nesse caso é um procedimento inadequado, uma vez que as conclusões finais obtidas se contradizem. De acordo com Santos et al. (1998), nos casos em que os fatores são de natureza quantitativa com mais de dois níveis, o emprego da análise de regressão é a alternativa mais apropriada e que, em termos gerais, consiste em ajustar uma função entre a resposta e os fatores em estudo.

TABELA 4: Peso de grãos (g) de feijão de acordo com diferentes doses de nitrogênio $\left(\mathrm{kg} \cdot \mathrm{ha}^{1}\right)$. Lages, 2007.

\begin{tabular}{cc}
\hline Doses & Peso \\
\hline 50 & $62.5917 \mathrm{a}$ \\
100 & $60.2500 \mathrm{~b}$ \\
0 & $50.2500 \mathrm{c}$ \\
\hline
\end{tabular}

*Médias seguidas de mesma letra não diferem entre si pelo teste de Tukey ao nível de significância de 5\%. 


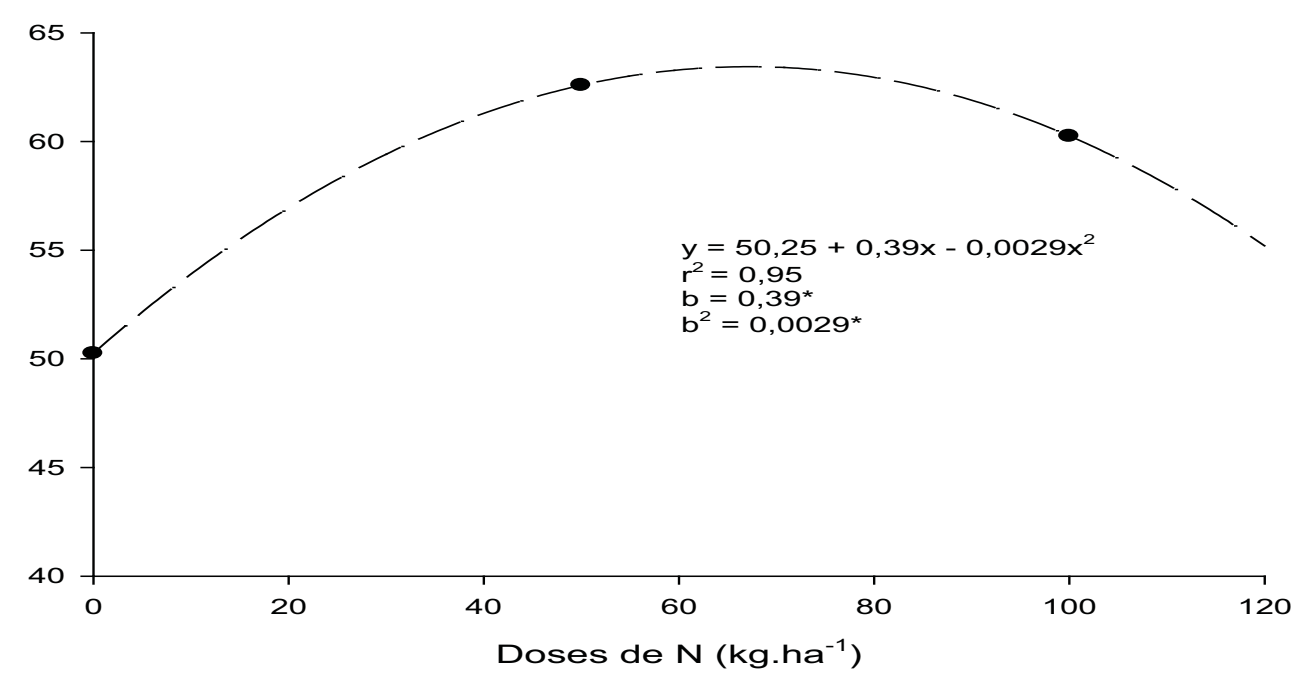

FIGURA 2: Peso de grãos de feijão em função de doses de nitrogênio.

Exemplo 2. Foi utilizado como segundo exemplo, um experimento com três tratamentos (areia, esterco e areia+esterco), classificado como qualitativo estruturado. Foi aplicado o teste de Tukey para a comparação de todos os pares de médias de tratamentos e contrastes ortogonais para a comparação entre grupo de médias. Os testes de comparação de médias, como o de Tukey, permitem ao pesquisador inferir sobre a média global dos tratamentos, ou seja, a comparação de todos os tratamentos entre si. Assim, em fatores qualitativos específicos estruturados, com combinações entre os tratamentos, somente é possível inferir sobre quais tratamentos obtiveram maior média, contrastando todos contra todos, onde o apropriado é a realização das comparações entre médias por contrastes ortogonais. De forma geral, os contrastes podem ser divididos em comparações, quando os efeitos de um grupo de tratamentos das variáveis em estudo são comparados com os de outro grupo (Alvarez e Alvarez, 2006).

Na tabela 5 estão demonstrados os resultados obtidos a partir do teste de Tukey, para os três tratamentos utilizados. Pode ser verificado que o tratamento que obteve maior média foi areia+esterco.
TABELA 5: Peso de grãos (g) mediante diferentes combinações de substratos. Lages, 2007.

\begin{tabular}{lc}
\hline \multicolumn{1}{c}{ Tratamentos } & Peso \\
\hline Areia & $41,00 \mathrm{~b}$ \\
Esterco & $42,00 \mathrm{~b}$ \\
Areia + Esterco & $55,00 \mathrm{a}$ \\
\hline
\end{tabular}

*Médias seguidas de mesma letra não diferem entre si pelo teste de Tukey, ao nível de significância de 5\%.

No entanto, essa conclusão é muito geral, relacionada com os efeitos do tratamento como um todo, não havendo informações específicas com relação ao componente responsável pelo incremento significativo no peso de grãos (g). Por meio de comparações através de contrastes ortogonais, pode ser afirmado qual componente que teve efeito significativo quando associado com outro componente. Por definição, um conjunto de contrastes é considerado ortogonal se todos os pares de contrastes do conjunto são ortogonais, ou seja, se a soma dos produtos dos correspondentes coeficientes dos dois contrastes é igual à zero. Os coeficientes inseridos na tabela 6 ilustram dois contrastes ortogonais de interesse do pesquisador. A ortogonalidade para os contrastes $C_{1}$ e $\mathrm{C}_{2}$ pode ser verificada por meio da soma do produto dos coeficientes $\mathrm{C}_{1}\left(-\begin{array}{lll}-2 & 1 & 1\end{array}\right) \times \mathrm{C}_{2}(0-11)$ pois $(2 \mathrm{x} 0)+$ $(1 \mathrm{x}-1)+(1 \mathrm{x} 1)=0$. 
Assim, realizando dois contrastes ortogonais (t1), pode ser verificado que a areia foi o componente do tratamento $\mathrm{A}+\mathrm{E}$ responsável pelo efeito significativo nas médias do tratamento areia+esterco (tabela 7).

TABELA 6: Conjunto ortogonal dos coeficientes para os contrastes dos três tratamentos.

\begin{tabular}{lccc}
\hline \multirow{2}{*}{ Contraste } & \multicolumn{3}{c}{ Tratamento } \\
\cline { 2 - 4 } & Areia (A) & Esterco (E) & $\mathbf{A}+\mathbf{E}$ \\
\hline $\mathrm{C}_{1}=\mathrm{A} v s \mathrm{E}, \mathrm{A}+\mathrm{E}$ & -2 & 1 & 1 \\
$\mathrm{C}_{2}=\mathrm{E}$ vs A+E & 0 & -1 & 1 \\
\hline Soma dos produtos & 0 & -1 & 1 \\
\hline
\end{tabular}

TABELA 7: Resumo da análise de variância e do desdobramento dos graus de liberdade por meio de contrastes ortogonais, para os tratamentos areia (A), esterco (E) e areia + esterco (A+E). Lages, SC.

\begin{tabular}{lccc}
\hline F.V. & G.L. & S.Q. & Q.M. \\
\hline Bloco & 2 & 16,66 & 8,33 \\
Substratos & $(2)$ & $366,00^{*}$ & $183,00^{*}$ \\
$\begin{array}{l}\text { Areia } v \text { esterco, } \\
\text { areia+esterco }\end{array}$ & 1 & $364,50^{*}$ & $364,50^{*}$ \\
$\begin{array}{l}\text { Esterco } v \text { s } \\
\text { areia+esterco }\end{array}$ & 1 & 1,50 & 1,50 \\
Erro & 4 & 65,33 & 16,33 \\
\hline Total & 8 & 448,00 & - \\
\hline
\end{tabular}

* Significativo ao nível de $5 \%$, pelo teste $F$.

Portanto, as dificuldades verificadas estão relacionadas aos experimentos fatoriais, onde há deficiência na decomposição dos graus de liberdades das interações e em experimentos unifatoriais, quanto ao uso inapropriado dos testes de comparação de médias em situações onde os tratamentos são de natureza quantitativa. A escolha inapropriada do teste adequado resulta em afirmações incompletas ou errôneas.

\section{Referências}

Aflakpui, G. K. S. 1995. Some uses/abuses of statistics in crop experimentation. Tropical Science, 35 (2): 347-353.

Alvarez, V. H.; Alvarez, G. A. M. 2006. Comparação de médias ou teste de hipóteses? Contrastes! Sociedade Brasileira de Ciências do Solo, 3 (1): 24-33.

Banzatto, D. A.; Kronka, S. N. 2006. Experimentação agrícola. 3. ed. FUNEP, Jaboticabal, Brasil, 247pp.

Bezerra Neto, F.; Nunes, G. H. S.; Negreiros, M. Z. 2002. Avaliação de procedimentos de comparações múltiplas em trabalhos publicados na revista Horticultura Brasileira de 1983 a 2000. Horticultura Brasileira, 20 (1): 5-9.

Cardellino, R. A.; Siewerdt, F. 1992. Utilização adequada e inadequada dos testes de comparação de médias. Revista da Sociedade Brasileira de Zootecnia, 21 (6): 985-995.

Chew, V. 1976. Comparing treatment means: a compendium. Hortscience, 11 (4): 348-357.

Gill, J. L. 1978. Design and analysis of experiments. v.1. Iowa State University Press, Ames, USA, 410pp.

Hinkelmann, K.; Kempthorne, O. 1994. Design and analysis of experiments: introduction to experimental design. JohnWiley, New York, USA, 495pp.

Jones, D. 1984. Use, misuse, and role of multiple comparison procedures in ecological and agricultural entomology. Environmental Entomology, 13 (3): 635-649.

Lúcio, A. D.; Lopes, S. J.; Storck, L.; Carpes, R. H; Lieberknecht, D.; Nicola, M. C. 2003. Características experimentais das publicações da ciência rural de 1971 a 2000. Revista Ciência Rural, 33 (1): 161-164.

Petersen, G. R. 1977. Use and misuse of multiple comparison procedures. Agronomy Journal, 69 (2): 205-208.

Santos, J. W.; Moreira, J. A. N.; Beltrão, N. E. M. 1998. Avaliação do emprego dos testes de comparação de médias na revista Pesquisa Agropecuária Brasileira (PAB) de 1980 a 1994. Pesquisa Agropecuária Brasileira, 33 (3): 225-230.

Silva, J. G. C. da. 1999. A consideração da estrutura das unidades em inferências derivadas do experimento. Pesquisa Agropecuária Brasileira, 34 (3): 911-925.

Spiegel, M. R. 1993. Estatística. $3^{\text {a }}$ ed. Makron Books, São Paulo, Brasil, 643pp.

Wechsler, F. S. 1998. Fatoriais fixos desbalanceados: uma análise mal compreendida. Pesquisa agropecuária Brasileira, 33 (3): 231-262.

Winer, B. J. 1971. Statistical principles in experimental design. $2^{\text {nd }}$ ed. McGraw-Hill, New York, USA, 907pp. 